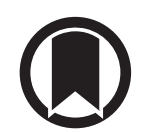

CrossMark

\title{
Upregulation of smooth muscle Rho-kinase protein expression in human asthma
}

\author{
To the Editor:
}

The lack of bronchodilatory response to deep inspiration in asthmatics is thought to be partially due to reduced airway distensibility $[1,2]$, possibly caused by an increase in airway smooth muscle (ASM) tone and stiffness [3]. Rho-kinase (ROCK) is known to play a role in regulating ASM tone [4] and ASM cytoskeletal stiffness [5].

ROCK is implicated in a wide range of diseases such as hypertension, arteriosclerosis and spinal cord injuries [6]. In pulmonary hypertension [7] and smokers [8], protein expression of both ROCK1 and ROCK2 is significantly increased in blood vessels and lung tissue. ROCK plays a critical role in angiogenesis by regulating endothelial cell migration and adhesion through actomyosin interaction and actin re-organisation, and by acting as mechano-transducer between integrin and the cytoskeleton. ROCK can also upregulate pro-angiogenic factors in the surrounding non-endothelial cells to maintain the angiogenic micro-environment [9]. Angiogenesis of bronchial microvessels is a feature of the pathology of asthma [10] and is associated with an augmented level of vascular endothelial growth factor, which has been shown to be released by ASM mechanical strain [11], such as the strain that occurs in asthma exacerbations. Elevated ROCK expression in blood vessels could further contribute to asthma-associated angiogenesis. When ROCK is inhibited pharmacologically [12] or knocked out genetically [13], airway hyperresponsiveness is abolished and ASM passive stiffness reduced [3]. An increase in the protein expression of ROCK in asthmatic ASM may contribute to exaggerated ASM contraction and stiffer airways. However, there has been no report to date on whether protein expression of ROCK is increased in the airways of asthmatics. Here, we report that the expression of both ROCK1 and ROCK2 is increased in ASM and pulmonary blood vessels in human asthma.

Age-matched non-asthmatic and asthmatic human lungs donated for research with consent through the International Institute of the Advancement of Medicine were used with approval from the University of British Columbia and St. Paul's Hospital Providence Health Care ethics committees. The diagnosis of asthma was based on the patients' medical records. Protein expression of both ROCK isoforms was quantified immunohistochemically on formalin-fixed paraffin embedded sections from trachea, main stem bronchi and parenchymal tissues from pre-inflated lungs. Standard immunohistochemical procedures were performed in a BOND-RX automated immunostaining machine (Leica Biosystems, Concord, ON, Canada). ROCK1 and ROCK2 isoforms were detected separately by the corresponding rabbit polyclonal antibody from the Prestige Antibodies series (Sigma Aldrich, St. Louis, MO, USA) at optimised concentrations: 1:100 and 1:200, respectively. Signals were detected by a biotin-free, polymeric alkaline phosphatase (AP)-linker antibody conjugate system optimised for the BOND-RX. The substrate chromogen, Fast Red, identified the antibody-antigen complex via a red precipitate. Haematoxylin counterstaining allowed visualisation of cell nuclei (blue). The slides were scanned with an Aperio ScanScope XT bright field scanner (Leica Biosystems), which allowed a maximal magnification of 40×, providing resolution of 0.25 microns.pixel ${ }^{-1}$. Using an image-viewer (Aperio ImageScope, Leica Biosystems), ASM and epithelium of each airway, each blood vessel embedded in the lung parenchyma, as well as each whole section (without distinguishing tissue type), were carefully encircled as regions of interest (ROI). Due to the limited maximal scanner resolution,

@ERSpublications

An important phenotypic change is associated with human asthma: the protein expression of both isoforms of Rho-kinase, ROCK1 and ROCK2, is increased in the smooth muscles of intraparenchymal airways and in the pulmonary blood vessels http://bit.ly/2pPfyxH

Cite this article as: Wang L, Chitano P, Paré PD, et al. Upregulation of smooth muscle Rho-kinase protein expression in human asthma. Eur Respir J 2020; 55: 1901785 [https://doi.org/10.1183/ 13993003.01785-2019]. 
a)
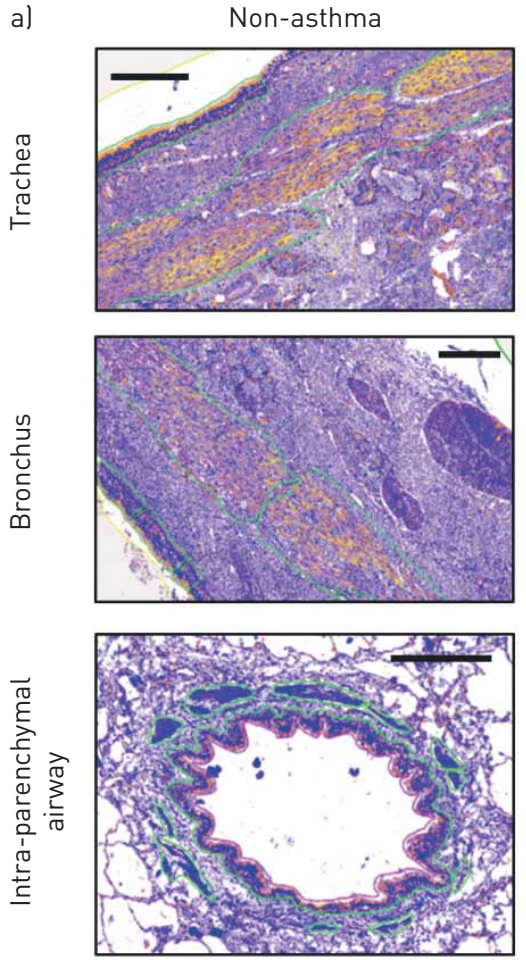

Negative Weak positive
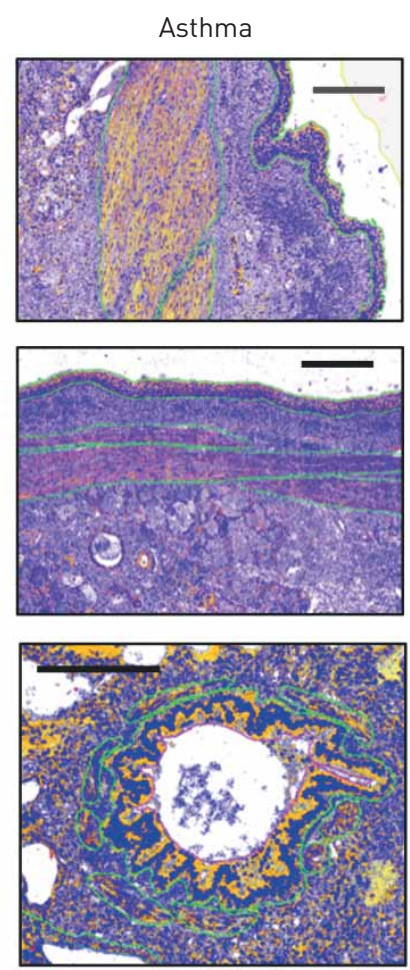

Positive b)
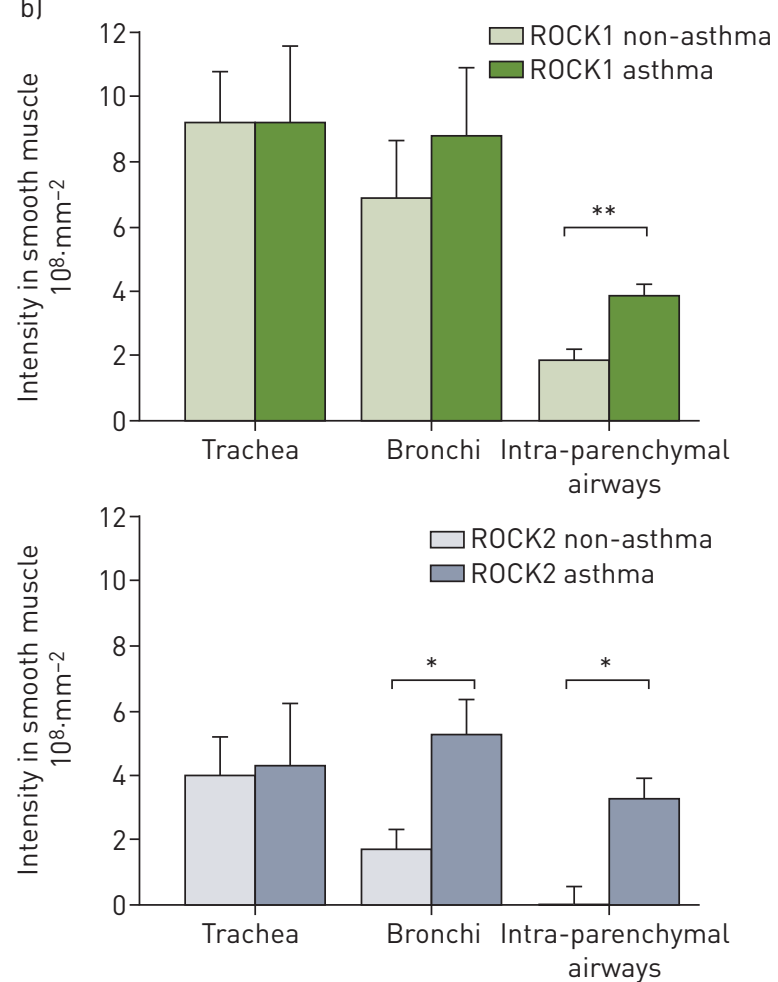

c)
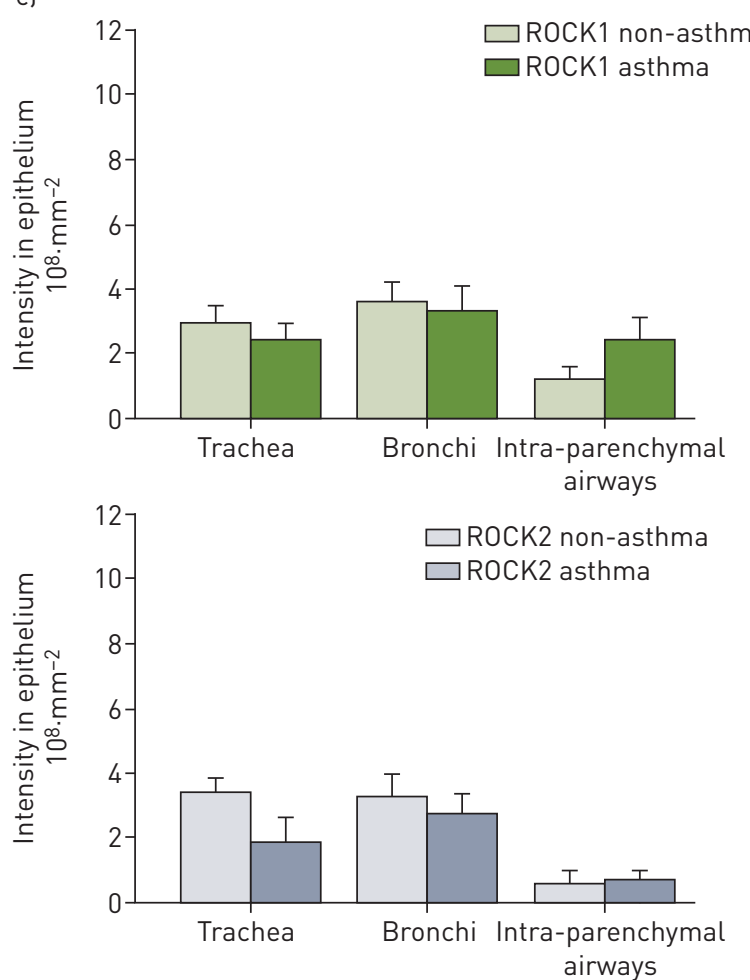

d)
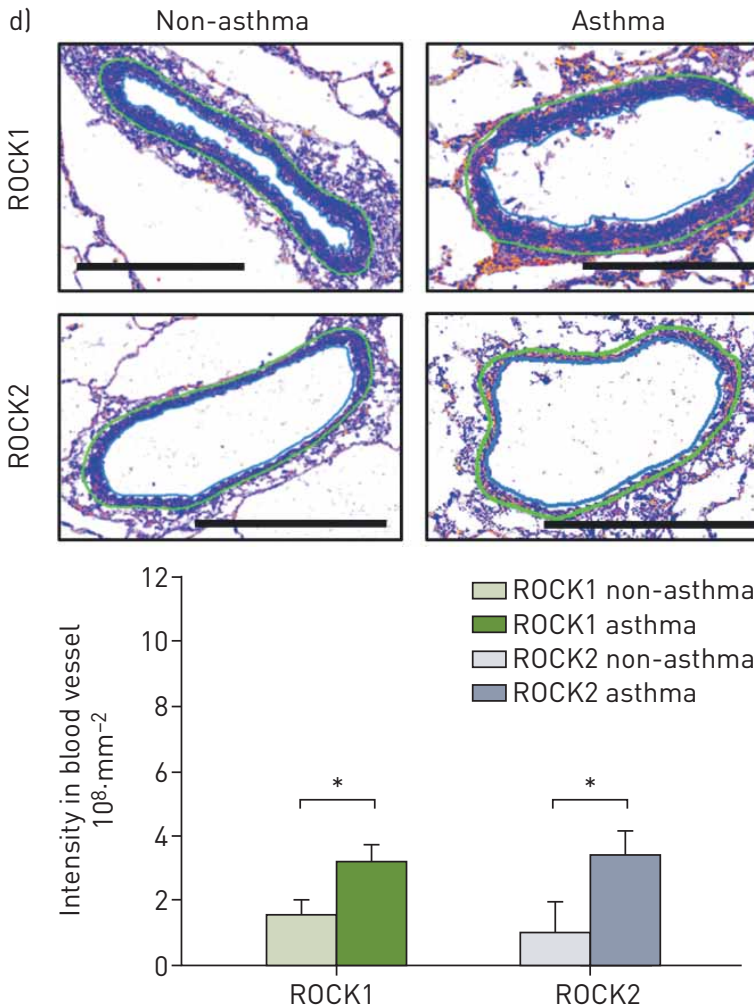

FIGURE 1 Rho-kinase (ROCK) protein expression is increased in the airways of asthma. a) Snapshots of ROCK1 staining in trachea, bronchi and intra-parenchymal airways; colour map shows intensity levels based on colour segmentation algorithms. b) Quantification of ROCK in airway smooth muscle (ASM). c) Quantification of ROCK in airway epithelium. d) Snapshots and quantification of ROCK1 and ROCK2 in blood vessels embedded in the lung parenchyma. Intensity is expressed as mean \pm SEM in arbitrary units $\left(10^{8} \cdot \mathrm{mm}^{-2}\right)$ as defined by the ImageScope software. Scale bars: $300 \mu \mathrm{m} .{ }^{*}:$ p $<0.05,{ }^{* *}: p<0.01$. 
we quantified the blood vessel ROCK expression in both endothelial and vascular smooth muscle layers as a whole. Colour segmentation algorithms were designed and applied to the ROI to detect positive staining. Non-specific staining was quantified from negative slides where primary antibody was omitted and was subtracted from the total staining intensity.

Four subjects with smoking history (two in each group) and one patient with a diagnosis of hypertension (non-asthma) were excluded from the analysis, resulting in eight non-asthmatic (six male and two female, all without respiratory disease or medication) and seven asthmatic subjects (three male and four female; two with mild, two with severe and three with fatal asthma). The non-asthmatic group was aged $28.4 \pm 14.9$ years (mean \pm SD); the asthmatic group aged $23.3 \pm 9.2$ years. One of the mild asthmatics used no medication and the other was on Adderall for attention deficit hyperactivity disorder. All severe and fatal asthmatics used daily inhalers containing steroids and bronchodilators. In addition, a severe asthmatic used the pain medication Oxycodone and a fatal asthmatic used allergy shots and Zyrtec. From preliminary tests of control samples, the standard deviation of average staining intensity was $20 \%$. Assuming that a $35 \%$ difference from the controls in the asthmatics would be a biologically meaningful difference, power calculations yielded a minimal sample size of 7 to detect a difference with a power of 0.8 $(\alpha=0.05)$. We first evaluated isoform distribution in non-asthmatics. In ASM and epithelium we performed two-way ANOVA, using isoform and location (trachea, bronchi and intra-parenchymal airways) as the two factors. In blood vessels and in whole lung sections, we performed t-tests to detect differences between isoforms. We then investigated whether ROCK expression was altered in asthmatics. In ASM and epithelium we performed two-way ANOVA using disease and location as the two factors. We performed t-tests to detect differences between asthma and non-asthma in blood vessels and whole lung sections.

ROCK protein expression is shown in figure 1 as staining intensity in arbitrary units, as defined by the ImageScope software, per unit area. Examples of colour segmentation images of ROCK1 staining in the airways and ROCK staining in the blood vessels are shown in figure 1a and d respectively. Both isoforms were expressed in greater quantities in trachea and bronchi than in intra-parenchymal airways for both ASM $(\mathrm{p}<0.001)$ and epithelium $(\mathrm{p}<0.001)$. ROCK2 staining was less than ROCK1 (ASM: $\mathrm{p}<0.001$, blood vessels: $\mathrm{p}=0.05)$. There was no difference between ROCK1 and ROCK2 in epithelium ( $\mathrm{p}=0.539)$. ROCK2 staining was less than ROCK1 in the whole lung sections $(\mathrm{p}=0.008)$. When comparing ROCK expression in non-asthmatics and asthmatics, we found that asthmatics had a significant increase in ASM ROCK1 ( $p=0.041)$ and ROCK2 $(\mathrm{p}=0.008)$ (figure $1 \mathrm{~b})$. The increase in ROCK1 occurred in intra-parenchymal airways $(\mathrm{p}<0.001)$, while ROCK2 was increased in bronchi $(p=0.015)$ and intra-parenchymal airways $(p=0.021)$. Neither isoform was increased in the trachea (ROCK1: $\mathrm{p}=0.791$, ROCK2: $\mathrm{p}=0.958$ ). In asthmatic epithelium (figure 1c), neither isoform increased significantly (ROCK1: $p=0.764$, ROCK2: $p=0.706$ ). In pulmonary blood vessels (figure $1 d$ ), both ROCK1 $(\mathrm{p}=0.017)$ and ROCK2 $(\mathrm{p}=0.014)$ were increased significantly in asthma. Both ROCK1 $(\mathrm{p}=0.003)$ and ROCK2 $(\mathrm{p}=0.044)$ were significantly increased in whole lung sections of asthmatics.

To our knowledge, this is the first evidence in human asthma that the protein expression of ROCK is elevated in intra-parenchymal airway- and vascular-smooth muscle, but not in tracheal smooth muscle. This is in agreement with our previous observation [14] that there is a general lack of difference in ASM mechanical properties in the trachea from asthmatics and non-asthmatic controls. Using trachea as a representative of all airways may in part underlie the inconsistencies in the literature about whether asthmatic ASM is intrinsically different from that of non-asthmatics. While ROCK is found to be increased, we have recently reported [15] that a RhoA/ROCK-inhibiting protein, p116 ${ }^{\text {Rip }}$, is reduced in the intra-parenchymal airways in asthma, leading to increased di-phosphorylation of myosin light chain, which could account for the observed increase in myosin filament stability in ASM [5]. Together, reduced p116 ${ }^{\text {Rip }}$ and increased ROCK, which are associated with reduced myosin light chain phosphatase function, mark a previously unknown phenotype in the ASM of asthma.

Although the functional consequences of anatomical differences in ROCK1 versus ROCK2 expression in asthma are still to be understood, upregulated ROCK expression in both airway and vascular smooth muscle in human asthma could be an important phenotypic change associated with the disease. Our finding supports the development of ROCK inhibitors for the treatment of asthma.

Lu Wang $\oplus^{1,2}$, Pasquale Chitano ${ }^{2,3}$, Peter D. Paré ${ }^{1,2}$ and Chun Y. Seow ${ }^{2,3}$

${ }^{1}$ Respiratory Division, Dept of Medicine, University of British Columbia, Vancouver, BC, Canada. ${ }^{2}$ Centre for Heart Lung Innovation, St. Paul's Hospital, University of British Columbia, Vancouver, BC, Canada. ${ }^{3}$ Dept of Pathology and Laboratory Medicine, University of British Columbia, Vancouver, BC, Canada.

Correspondence: Lu Wang, UBC Centre for Heart Lung Innovation, St. Paul's Hospital, 1081 Burrard Street, Rm 166, Vancouver, BC, Canada, V6Z 1Y6. E-mail: lu.wang@hli.ubc.ca

Received: 11 Sept 2019 | Accepted after revision: 4 Nov 2019 
Conflict of interest: L. Wang reports grants from Canadian Institutes of Health Research, Natural Sciences and Engineering Research Council of Canada and British Columbia Lung Association, during the conduct of the study. P. Chitano reports grants from Canadian Institutes of Health Research, Natural Sciences and Engineering Research Council of Canada and British Columbia Lung Association, during the conduct of the study. P.D. Paré reports grants from Canadian Institutes of Health Research, Natural Sciences and Engineering Research Council of Canada and British Columbia Lung Association, during the conduct of the study. C.Y. Seow reports grants from Canadian Institutes of Health Research, Natural Sciences and Engineering Research Council of Canada and British Columbia Lung Association, during the conduct of the study.

Support statement: This work was funded by the Canadian Institutes of Health Research, Natural Sciences and Engineering Research Council of Canada, and the British Columbia Lung Association. Funding information for this article has been deposited with the Crossref Funder Registry.

\section{References}

1 Colebatch HJ, Finucane KE, Smith MM. Pulmonary conductance and elastic recoil relationships in asthma and emphysema. J Appl Physiol 1973; 34: 143-153.

2 Pyrgos G, Scichilone N, Togias A, et al. Bronchodilation response to deep inspirations in asthma is dependent on airway distensibility and air trapping. J Appl Physiol 2011; 110: 472-479.

3 Raqeeb A, Jiao Y, Syyong HT, et al. Regulatable stiffness in relaxed airway smooth muscle: a target for asthma treatment? J Appl Physiol 2012; 112: 337-346.

4 Puetz S, Lubomirov LT, Pfitzer G. Regulation of smooth muscle contraction by small GTPases. Physiology (Bethesda) 2009; 24: 342-356.

5 Lan B, Deng L, Donovan GM, et al. Force maintenance and myosin filament assembly regulated by Rho-kinase in airway smooth muscle. Am J Physiol Lung Cell Mol Physiol 2015; 308: L1-L10.

6 Loirand G. Rho kinases in health and disease: from basic science to translational research. Pharmacol Rev 2015, 67: 1074-1095.

7 Do.e Z, Fukumoto Y, Takaki A, et al. Evidence for Rho-kinase activation in patients with pulmonary arterial hypertension. Circ J 2009; 73: 1731-1739.

8 Duong-Quy S, Dao P, Hua-Huy T, et al. Increased Rho-kinase expression and activity and pulmonary endothelial dysfunction in smokers with normal lung function. Eur Respir J 2011; 37: 349-355.

9 Liu J, Wada Y, Katsura M, et al. Rho-associated coiled-coil kinase (ROCK) in molecular regulation of angiogenesis. Theranostics 2018; 8: 6053-6069.

10 Harkness LM, Kanabar V, Sharma HS, et al. Pulmonary vascular changes in asthma and COPD. Pulm Pharmacol Ther 2014; 29: 144-155.

11 Hasaneen NA, Zucker S, Lin RZ, et al. Angiogenesis is induced by airway smooth muscle strain. Am J Physiol Lung Cell Mol Physiol 2007; 293: L1059-L1068.

12 Schaafsma D, Bos IS, Zuidhof $\mathrm{AB}$, et al. The inhaled Rho kinase inhibitor Y-27632 protects against allergen-induced acute bronchoconstriction, airway hyperresponsiveness, and inflammation. Am J Physiol Lung Cell Mol Physiol 2008; 295: L214-L219.

13 Kasahara DI, Ninin FM, Wurmbrand AP, et al. Abrogation of airway hyperresponsiveness but not inflammation by rho kinase insufficiency. Clin Exp Allergy 2015; 45: 457-470.

14 Chin LY, Bossé Y, Pascoe C, et al. Mechanical properties of asthmatic airway smooth muscle. Eur Respir J 2012; 40: 45-54.

15 Komatsu S, Wang L, Seow CY, et al. p116 ${ }^{\text {Rip }}$ promotes myosin phosphatase activity in airway smooth muscle cells. J Cell Physiol 2020; 235: 114-127. 